\title{
Fat Myringoplasty and its Impact on the Hearing Mechanism of Middle Ear
}

\author{
1Bandar Al-qahtani, ${ }^{2}$ Mohammed Al Tuwaijri, ${ }^{3}$ Mohammed Al Mokhatrish
}

\begin{abstract}
Objectives: To address the fat grafting to tympanic membrane perforations - fat myringoplasty type I-and its impact over the hearing mechanism of the middle ear for both small $(<25 \%$ of the tympanic membrane) and large perforation (>75\% of the TM).
\end{abstract}

Keywords: Closure of air-bone gap, Fat graft, Tympanoplasty.

How to cite this article: Al-qahtani B, Al Tuwaijri M, Al Mokhatrish M. Fat Myringoplasty and its Impact on the Hearing Mechanism of Middle Ear. Int J Otorhinolaryngol Clin 2015;7(3):138-140.

Source of support: Nil

Conflict of interest: None

\section{INTRODUCTION}

Myringoplasty is an operative procedure used in the reconstruction of a perforated tympanic membrane. ${ }^{1}$ It is one of the most common surgical procedure in otology. The term myringoplasty was named by Berthold in 1878, but the first myringoplasty was performed by Marcus Bancer in 1640. ${ }^{1}$ In 1952, Wullstein published a method of closing perforations with a split thickness skin graft. One year later Zollner reported his experiences with a similar graft; Wullstein and House then advised a full thickness graft taken from behind the ear. In 1961, Storrs used temporal fascia to close tympanic membrane perforation. During the last three decades, temporal fascia has been the most commonly used graft in myringoplasty operations, although different grafts were also used to repair a tympanic membrane perforations. A spectrum of grafts, the most common are auto grafts (from the patient himself like perichondrium, fat, cartilage, etc.), homo grafts (from different patient like cartilage, dura), and xenografts or heterografts (from other species other than humans like pigs, monkeys, tragal perichondrium, bovine pericardium, etc.). ${ }^{2,3}$

\footnotetext{
${ }^{1,2}$ Consultant, ${ }^{3}$ Resident

${ }^{1-3}$ Department of Otorhinolaryngology, King Saud Medical City Riyadh, Saudi Arabia
}

Corresponding Author: BandarAl-qahtani, Consultant, Department of Otorhinolaryngology, King Saud Medical City, Riyadh Saudi Arabia, Phone: 00966555646405, e-mail: khamsi20@ yahoo.com
The main goal or aim of the ear surgery has been directed toward the restoration of function as well as providing a stabilized trouble-free ear. ${ }^{4,5}$ Zollner and Wullstein provided a classification of tympanoplasty that focused on the type of ossicular chain reconstruction (OCR) needed. The five types of tympanoplasty they described refer to the most lateral intact structure on which the conductive mechanism will be constructed. Type I tympanoplasty indicates all three ossicles to be present and mobile. Thus, OCR is not needed. Type II grafts the tympanic membrane to an intact incus and spates. A type III exists when an intact mobile spates superstructure is present and the tympanic membrane or graft remains directly on the spates superstructure. Type IV describes an absent or eroded superstructure with the graft or tympanic membrane overlying a mobile spates footplate. Type $\mathrm{V}$ tympanoplasty refers to a fenestration created in the horizontal semicircular canal. ${ }^{4,5}$

The reasons for tympanoplasty are to eliminate recurrent disease, provide a dry ear canal and middle ear space with an intact tympanic membrane, and improve hearing. This includes perforation or hearing loss due to trauma, infection, or prior surgery that persists for more than 3 months. Inability to safely bathe or participate in water activities because of perforation of the tympanic membrane, with or without hearing loss is also an indication. Ringenberg was the first one who reported the use of fat as a graft in tympanoplasty, success rate of repairing of the perforation ranged between 76 and 100\% reported by a lot of study ever since. ${ }^{6}$ Fat myringoplasty (FM), or myringoplasty with a fat graft, can be done in the standard otosurgical position and draped via sterile technique and usually can be done under local anesthesia. Fat graft is easily obtained from the ear lobules of the operated ear as well as from abdominal fat. ${ }^{7}$

\section{DESIGN}

Review of literatures between June 2010 and June 2014.

\section{RESULTS AND DISCUSSION}

The middle ear transforms acoustic energy from the medium of air to the medium of fluid. ${ }^{4,5}$ It is an impedancematching system that ensures that energy is not lost. This impedance matching is accomplished by the area effect 
of the tympanic membrane and the lever action of the ossicular chain. Although the area of the adult tympanic membrane is between 85 and $90 \mathrm{~mm}^{2}$ only about $55 \mathrm{~mm}^{2}$ effectively vibrates (the lower $2 / 3$ of the drum); the stapes footplate is $3.2 \mathrm{~mm}^{2}{ }^{6,8}$ Thus, the ratio of the vibrating portion of the tympanic membrane to that of the stapes footplate results in a 17:1 increase in sound energy. Also, because the handle of the malleus is approximately 1.3 times longer than the incus long process, the force (pressure) received at the stapes footplate, through the use of leverage, is greater than that at the malleus by about 1.3:1. Thus, the combined transformer ratio of the middle ear is about 22:1 $(17 \times 1.3=22)$. This translates to approximately $25 \mathrm{~dB} .6,8,10$

A simple perforation of the tympanic membrane without other lesions of the middle ear transformer has two different effects on the hearing. First, there is the diminished surface of tympanic membrane on which sound pressure is exerted, causing diminished excursions of the ossicular chain. The second effect on the hearing of a simple perforation results from sound reaching the round window directly without the dampening and phase-changing effect of the intact tympanic membrane. This effect becomes greater with a larger perforation, so that sound reaches both windows with more nearly equal force and at nearly the same time. The resultant cancellation of vibratory movement of the cochlear fluid column produces the maximum hearing loss observed in simple perforation, as much as $42 \mathrm{~dB}$ for the speech frequencies.

Fat myringoplasty can be performed under local anesthesia as an office procedure. ${ }^{11,14}$ It is a relatively safe procedure because the likelihood of otologic trauma from the manipulation of the tympanic cavity is reduced. Bilateral surgery is also possible, and the postoperative care required is minimal. In FM, the closure rates for small perforations were higher than those for large perforations ( 93.7 vs $70.7 \%$ ), and the posterior perforations had better closure rates $(90.5 \%)$ than did anterior perforations $(67.7 \%)$. The results of FM in revision cases were poor $(52.9 \%) .{ }^{8}$ The effects of the size, site, and status of the remnant membrane and the cause of its perforation play major role on surgical outcome of FM. The size of the perforation was determined by several parameters: the percentage of the pars tensa involved, ${ }^{6,9,10}$ the number of involved quarters of the tympanic membrane, ${ }^{7}$ the diameter of the perforation, and the gross dimension of the perforation. ${ }^{11}$ Only a few authors used FM in revision surgery. ${ }^{12,13}$ The size of the perforation is the main criterion used by many investigators to select candidates for FM. According to Kaddour HS, the size of the perforation should not exceed $30 \%$ of the size of the eardrum, closure rate, $80 \% .{ }^{14}$ Terry et al, who performed FM to correct perforations of various sizes, cited a closure rate of $79.4 \%$ if the perforation accounted for less than $50 \%$ of tympanic membrane and $57.1 \%$ if the perforation was larger than that size. ${ }^{9}$ Bertoli et al, found a low closure rate for anterior perforations $(67.7 \%$ with no statistically significant difference between groups small and large perforations). In posterior perforations, the overall closure rate was $90.5 \%$, and $100 \%$ of the patients with small perforation. ${ }^{8}$ In anterior perforation, the anterior edge of the perforation is usually partially obscured by the bulging of the anterior ear canal. As a result, the scarred margins may not be adequately refreshed leading to poor results. In such cases, Ayache et al suggest the use of otoendoscopy for better visualization of the anterior margin. ${ }^{7}$ Also, the success of closure was correlated with the cause of the perforation. It is noticed in patients with stapes surgery whose perforation was caused by an iatrogenic injury, the percentage of perforation closure was significantly high (perhaps because of the normal vascular supply and intact vascular strip). However, this can explain the poor results of revision surgery of the ear beside the Eustachian tube problems, ear infection, poor blood supply in the eardrum, etc. Despite those poor results in that group of patients, FM can be advisable to those with reperforation of the eardrum post tympanoplasty (e.g. revision cases or on a patient who refuses to return to the operating theater). ${ }^{8}$

In other studies, perforations that were not larger than a quadrant of the pars tensa showed an overall success rate reaching $80.8 \% .{ }^{8}$ In patients with a small perforation, the closure ratio was $93.7 \%$, and in patients with a larger perforation, the closure rate was $70.7 \%$. To emphasize that the perforation size increased in all patients after the removal of the edges of the perforation, so the selection criterion of size (less than one-fourth of the pars tensa) could be. ${ }^{5,6,8}$

Presence of myringosclerosis or atrophy of tympanic membrane also affect the surgical outcomes. In FM, the size of the graft that 2 or 3 times larger than the perforation can be essential and necessarily.

Therefore, infiltration of an anesthetic could artificially enlarge the volume of fat tissue in the ear lobe and underestimating, although the excised fat may seems sufficient to close the perforation after dehydration and may become too small to close the perforation. As FM is not difficult procedure; however, performing FM is not without challenges. Finding a graft that is large enough to repair the perforation in patients with a small lobule can be difficult. The possibility of postsurgical lobule deformity exists, ${ }^{8}$ as does the theoretical implantation of the skin debris in the graft and the consequent development of 
an iatrogenic secondary cholesteatoma. ${ }^{8,9,11}$ A sense of fullness in the ear and temporary tinnitus, which are probably caused by the long-term persistence of the graft block, can develop. Some patients complain of an auricular discharge, which may be melting fat, for a few weeks after surgery. 8,14

\section{CONCLUSION}

Fat-graft myringoplasty (FGM) has a reliable tympanic closure rate for small to large perforations and it seems that it has a better hearing results in closure of small TM perforation than large TM perforation. As in different studies, no significant impact on ossicles movement, and hence hearing mechanism. Apart from feeling a sense of fullness, topographic evaluation of FGM showed that the procedure resulted in a reliable perforation closure rate and good audiologic outcome, regardless of perforation locations.

\section{REFERENCES}

1. Shambaugh and Glasscock. Surgery of the Ear. 1980. p. 411-412.

2. Gibb AG, Chang SK. Myringoplasty: a review of 365 operations. J Laryngol Otol 1982;96:915-930.

3. Downey TJ, Champeaux AL, Silva AB. AlloDerm tympanoplasty of tympanic membraine perforation. Am J Otolaryngol 2003;24:6-13
4. Sheehy JL, Shelton C. Tympanoplasty: to stage or not to stage. Otolaryngol Head Neck Surg 1991 Mar;104(3):399-407.

5. Hirsch BE. Myringoplasty and tympanoplasty. In: Myers EN, editor. Operative Otolaryngology/Head and Neck Surgery. 2nd ed. Saunders; 2008. p. 1246-1261.

6. Ringenberg JC. Fat graft tympanoplasty. Laryngoscope 1962;72:188-192.

7. Ayache S, Braccini F, Facon F, Thomassin JM. Adipose graft: an original option in myringoplasty. Otol Neurotol 2003; 24:158-164.

8. Bertoli GA, et al. Fat graft myringoplasty: an office procedure for the repair of small perforations of the tympanic membrane. The Mediterranean J Otology 2007;3:120-125.

9. Terry RM, Bellini MJ, Clayton MI, Gandhi AG. Fat graft myringoplasty-a prospective trial. Clin Otolaryngol Allied Sci 1988;13:227-229.

10. Deddens AE, Muntz HR, Lusk RP. Adipose myringoplasty in children. Laryngoscope 1993;103:216-219.

11. De S, Karkanevatos A, Srinivasan VR, Roland NJ, Lesser TH Myringoplasty using a subcutaneous soft tissue graft. Clin Otolaryngol Allied Sci 2004;29:314-317.

12. Ozgursoy OB, Yorulmaz I. Fat graft myringoplasty: a cost-effective but underused procedure. J Laryngol Otol 2005;119:277-279.

13. Liew L, Daudia A, Narula AA. Synchronous fat plug myringoplasty and tympanostomy tube removal in the management of refractory otorrhoea in younger patients. Int J Pediatr Otorhinolaryngol 2002;66:291-296.

14. Kaddour HS. Myringoplasty under local anaesthesia: day case surgery. Clin Otolaryngol Allied Sci 1992;17:567-568. 